\title{
Esquemas terapéuticos y factores asociados a mortalidad en pacientes con cuadro severo de COVID-19 atendidos en Hospital Nacional Alberto Sabogal Sologuren 2020
}

\author{
Manuel K Llaro-Sánchez 1,a; Ronald N Guzman-Ramos 1,b; Bernardo E Gamarra-Villegas 2,c; Karen E Campos-Correa* 3,d
}

RESUMEN

Objetivo: Explorar el esquema terapéutico y factores asociados a mortalidad en pacientes con un cuadro severo de COVID-19 atendidos en el Hospital Nacional Alberto Sabogal Sologuren en 2020.

Materiales y métodos: Estudio observacional, analítico de caso-control y prospectivo. Fueron incluidos los pacientes que ingresaron al hospital con un cuadro severo de COVID-19 entre junio a septiembre de 2020 y fueron clasificados en dos grupos: el grupo caso (61 pacientes fallecidos) y el grupo control (60 sobrevivientes que recibieron el alta hospitalaria). Los datos fueron analizados en el software estadístico $S_{t a t a}{ }^{(R)}$ y se realizó análisis bivariado y multivariado con regresión logística, nivel de confianza del $95 \%$.

Resultados: En pacientes con cuadro severo de COVID-19, la edad mayor de 60 años está asociada a la mortalidad ( $p=0,035$; OR=2,21 IC: $[1,05-4,63])$. Los esquemas terapéuticos fueron variados, los participantes que recibieron metilprednisolona a dosis altas tuvieron mayor probabilidad de morir en comparación a los que recibieron otros corticoides $(p=0,001 ;$ OR ajustado=5, 18 IC: $[1,94-13,83])$. El tratamiento con azitromicina por más de cinco días incrementa la probabilidad de fallecer en comparación a los que la tomaron por menos días ( $p=0,000$; OR ajustado=7,14 IC: [2,22-22,99]). El modelo multivariado tenía una probabilidad predictiva de mortalidad de 73,06 \% para los pacientes con cuadro severo de COVID-19.

Conclusiones: Los esquemas terapéuticos que incluyen administración de metilprednisolona a dosis altas y azitromicina por más de 5 días incrementan la probabilidad de fallecer en los pacientes con una presentación severa de COVID-19. Además, la edad mayor a 60 años estuvo asociada a la mortalidad en los pacientes analizados en el estudio.

Palabras clave: Infecciones por coronavirus; Síndrome respiratorio agudo grave; Terapéutica; Corticoesteroides; Mortalidad (DeCS BIREME).

\section{Therapeutic regimens and factors associated with mortality in patients with severe COVID-19 infection treated at the Hospital Nacional Alberto Sabogal Sologuren, 2020}

\section{ABSTRACT}

Objective: To explore the therapeutic regimen and factors associated with mortality in patients with severe COVID-19 infection treated at the Hospital Nacional Alberto Sabogal Sologuren in 2020.

Materials and methods: An observational, case-control, analytical and prospective study conducted in patients with severe COVID-19 infection at hospital admission between June and September 2020. The study population was classified into two groups: case group (61 deceased patients) and control group (60 discharged patients). Data were analyzed using Stata statistical software. Bivariate and multivariate logistic regression analyses were performed with a $95 \%$ confidence level.

Results: As for patients with severe COVID-19 infection, ages older than 60 years are associated with mortality $(p=0.035 ;$ OR $=2.21$; $\mathrm{Cl}$ : 1.05 - 4.63]). Different therapeutic regimens were included in the research: patients who received high-dose methylprednisolone had more probability of dying compared to those who received other corticosteroids ( $p=0.001$; adjusted OR = 5.18; Cl: [1.94 13.83]). Patients who were treated with azithromycin for more than five days had more probability of dying compared to those that took it for fewer days ( $p=0.000$; adjusted $\mathrm{OR}=7.14 ; \mathrm{Cl}$ : [2.22 - 22.99]). The multivariate model showed a 73.06 \% predictive probability of mortality for patients with severe COVID-19 infection.

Conclusions: The therapeutic regimens that include the administration of high-dose methylprednisolone and azithromycin for more than five days increase the probability of dying in patients with severe COVID-19 infection. Furthermore, ages over 60 years were associated with mortality in the patients who participated in the study.

Keywords: Coronavirus infections; Severe acute respiratory syndrome; Therapeutics; Adrenal cortex hormones; Mortality (Source: MeSH NLM).

1 Hospital Nacional Alberto Sabogal Sologuren. Lima, Perú.

2 Instituto de Medicina Legal y Ciencias Forenses. Lima, Perú.

3 Universidad Peruana Cayetano Heredia. Lima, Perú.

a Médico Especialista en Nefrología, Egresado de la Maestría en Epidemiología clínica.

b Médico Especialista en Enfermedades Infecciosas y Tropicales, Egresado de Maestría en Medicina.

c Médico Cirujano, Egresado de Maestría en Epidemiología clínica.

d Licenciada en Obstetricia, Egresada de Maestría en Epidemiología clínica.

* Autor corresponsal.

Horiz Med (Lima) 2021; 21(1): e1346 


\section{INTRODUCCIÓN}

A finales de 2019 aparece en China un nuevo coronavirus, denominado SARS-CoV-2 ${ }^{(1)}$, que se expandió a más de 114 países (2). En el Perú, el segundo país más golpeado en Latinoamérica (3), al 3 de enero de 2021, 1019475 casos estaban confirmados y habían fallecido 37830 personas ${ }^{(4)}$. El $26 \%$ de los pacientes presenta un cuadro clínico severo $y^{(5)}$, de ellos, fallece el $45,4 \%{ }^{(6)}$.

Los estudios no demuestran una terapia clínica eficaz y concluyente para el manejo de la COVID-19. Así, Xu et al. refieren que el tocilizumab es efectivo para reducir mortalidad (7); Mahevas et al. no encontraron evidencia de eficacia clínica de hidroxicloroquina ${ }^{(8)}$. Por otro lado, el Ministerio de Salud incorporó los corticoides y la enoxaparina en el esquema terapéutico ${ }^{(9)}$. Por tanto, la búsqueda del tratamiento más efectivo y seguro es intensa. Como factores que incrementan el riesgo de mortalidad, $\mathrm{Li}$ et al. refieren al sexo masculino (HR:1,7), edad mayor de 65 años (HR:1,7) ${ }^{(10)}$; así mismo, durante la hospitalización observaron injuria cardiaca (HR: 2,9), hiperglicemia (HR:1,8) y altas dosis de corticoides administrados (HR:3,5). Zhou et al., encontraron resultados similares y también reportan que la hipertensión arterial es la comorbilidad más frecuente en los pacientes fallecidos $(\mathrm{OR}: 3,05)^{(6)}$.

En el Perú existe la necesidad de investigar y generar evidencias sobre los resultados terapéuticos y factores que determinan el riesgo de mortalidad. Por esta razón, es prioritario identificar cuáles son las intervenciones terapéuticas que brindan los mejores resultados para el manejo de la COVID-19.

Por tanto, la presente investigación busca explorar el esquema terapéutico y los factores asociados a la mortalidad en pacientes con un cuadro severo de COVID-19 atendidos en el Hospital Nacional Alberto Sabogal Sologuren.

\section{MATERIALES Y MÉTODOS}

\section{Diseño y población de estudio}

Investigación de tipo observacional, analítica de caso-control y prospectiva. La población estuvo conformada por los pacientes que ingresaron al Hospital Nacional Alberto Sabogal Sologuren con un cuadro severo de COVID-19 durante los meses de junio a septiembre de 2020. La clasificación de severidad fue hecha según la Guía del Ministerio de Salud ${ }^{(11)}$. La muestra se calculó en programa OpenEpi v.3.0, fórmula de casos y controles no pareados, con una potencia del $80 \%$ y nivel de confianza del $95 \%$. El factor utilizado para el cálculo de la muestra fue la presencia de la hipertensión arterial, ya que diversos estudios coindicen en que es un factor importante asociado a la mortalidad y severidad en los enfermos con COVID-19 ${ }^{(6,10)}$. La proporción del factor en el grupo control $(23 \%)$ y en el grupo caso (48\%) fue tomada de la investigación de Zhou et al. ${ }^{(6)}$.
La muestra quedó conformada por 121 pacientes con cuadro severo de COVID-19: 61 fallecidos durante la hospitalización (grupo caso) y 60 enfermos que sobrevivieron y recibieron el alta hospitalaria (grupo control). Los pacientes adultos de ambos sexos con estudio confirmatorio de COVID-19, mediante prueba rápida o prueba molecular, fueron incluidos en la investigación. Los enfermos referidos a otros hospitales o con altas voluntarias no participaron en el estudio. Todos los pacientes tenían las características radiológicas compatibles con COVID-19, que fueron más frecuentes en los hombres $(75,00 \%)$ que en las mujeres $(25,00 \%)$. El número de fallecidos y sobrevivientes fue similar. La edad promedio de los participantes fue de 62,98 \pm 14 años y la única causa de muerte reportada fue insuficiencia respiratoria aguda.

\section{Variables y mediciones}

Los datos se recolectaron de la historia clínica del paciente al salir de alta. Las variables independientes fueron esquemas de tratamiento, características epidemiológicas, comorbilidades, asistencia por ventilación mecánica, y la variable dependiente es la mortalidad. Todas ellas se tomaron de investigaciones previas y del Documento Técnico del Ministerio de Salud para COVID-19 en Perú (11) y sus modificaciones ${ }^{(9,12,13)}$. La información de la historia clínica fue anotada en una ficha de recolección de datos física (Anexo 1), a la que se colocó un número para evitar la identificación del paciente. En un listado aparte quedó registrada la historia clínica relacionada con el número de la ficha de datos, este documento fue de acceso exclusivo del equipo investigador.

\section{Análisis estadístico}

Los datos fueron analizados en el software estadístico Stata $^{(R)}$ 16.0. Para la comparación de variables numéricas con distribución normal se utilizó la prueba t de Student y para las variables sin distribución normal, la prueba U-Mann Whitney. En las variables categóricas, la asociación se analizó con la prueba de $X^{2}$ de Pearson o la prueba exacta de Fisher. Empleamos Odds Ratio (OR) para calcular la fuerza de asociación, y reportamos el OR crudo y ajustado según los siguientes factores: edad del paciente y presencia de alguna comorbilidad. Finalmente, se realizó análisis multivariado de regresión logística por anidamiento con nivel de confianza al $95 \%$.

\section{Consideraciones éticas}

La investigación fue aprobada por el Comité de Ética en Investigación Específico para COVID-19 de EsSalud. El consentimiento informado no fue necesario porque los datos se recolectaron de una fuente secundaria digital, sin contacto con pacientes. Por respeto a la confidencialidad de los participantes, los miembros del equipo investigador fueron los únicos que custodiaron y manejaron la lista de códigos con la información de los pacientes. Los principios bioéticos de investigación en seres humanos fueron respetados, no hubo discriminación por causa de raza, 
sexo, procedencia, etc. Los pacientes y los recolectores de datos no estuvieron expuestos a algún tipo de riesgo.

\section{RESULTADOS}

Entre las características epidemiológicas encontramos que la edad promedio en los fallecidos fue mayor $(66,27 \pm 12,24)$ que en los sobrevivientes $(59,63 \pm 15,08)$, con diferencia estadísticamente significativa $(p=0,008)$. La edad mayor de 60 años se asoció a mortalidad (OR=2,21 IC:1,05-4,63). No hubo asociación entre presencia de comorbilidades y mortalidad. Las comorbilidades más frecuentes fueron hipertensión arterial, diabetes y obesidad. El tiempo de enfermedad prehospitalario entre fallecidos (Me:10 días RIQ 3) y sobrevivientes (Me:8,5 días RIQ 4,5) fue similar $(p=0,874)$.
El $40,98 \%$ de pacientes fallecidos y el $33,33 \%$ de sobrevivientes reportaron haber tomado medicamentos previamente, entre ellos, azitromicina, ivermectina y corticoides.

Los esquemas terapéuticos administrados fueron variados, en todos ellos emplearon corticoides y en casi todos, enoxaparina. Alrededor del $60,00 \%$ de los pacientes recibieron azitromicina $y$, en menor frecuencia, hidroxicloroquina, ivermectina y tocilizumab. La ceftriaxona fue el antibiótico parenteral más utilizado. Hallamos diferencias significativas entre la frecuencia de uso de corticoides en pacientes que fallecieron y los que sobrevivieron $(p=0,004)$ (Tabla 1$)$.

Tabla 1. Esquemas terapéuticos y mortalidad en pacientes con cuadro severo de COVID-19 en el Hospital Nacional Alberto Sabogal Sologuren

\section{Esquemas terapéuticos}

\section{Mortalidad}

sí

$\%$

6,56

6,56

8,33

$0,743^{a}$

No

57

93,44

93,44

91,67

Azitromicina

Sí

No

Enoxaparina

$$
\text { Sí }
$$

No

41

67,21

32,79

\section{7,21}

32,79

100,0

0
100,0

0

60,00

40,00
$\%$ p

\begin{tabular}{|c|c|c|c|c|c|}
\hline \multirow[t]{2}{*}{ Tiempo de enoxaparina (días) } & $\mathrm{N}=61$ Mediana & RIQ & RIQ & RIQ & \\
\hline & 8 & 5 & 5 & 20 & $0,003^{c}$ \\
\hline \multicolumn{6}{|l|}{ Ivermectina } \\
\hline Sí & 7 & 11,48 & 11,48 & 3,33 & $0,163^{\mathrm{a}}$ \\
\hline No & 54 & 88,52 & 88,52 & 96,67 & \\
\hline \multicolumn{6}{|l|}{ Tocilizumab } \\
\hline Sí & 1 & 1,64 & 1,64 & 5,0 & $0,365^{\mathrm{a}}$ \\
\hline No & 60 & 98,36 & 98,36 & 95,0 & \\
\hline \multicolumn{6}{|l|}{ Corticoides } \\
\hline Dexametasona & 37 & 60,66 & 60,66 & 73,32 & $0,004^{b}$ \\
\hline Metilprednisolona & 23 & 37,70 & 37,70 & 11,67 & \\
\hline Prednisona & 0 & 0 & 0 & 1,67 & \\
\hline Hidrocortisona & 0 & 0 & 0 & 1,67 & \\
\hline Dexametasona+ Metilprednisolona & 1 & 1,64 & 1,64 & 11,67 & \\
\hline
\end{tabular}




\begin{tabular}{|c|c|c|c|c|c|}
\hline \multirow{3}{*}{ Esquemas terapéuticos } & \multicolumn{5}{|c|}{ Mortalidad } \\
\hline & \multicolumn{2}{|c|}{ sí } & \multicolumn{2}{|c|}{ NO } & \multirow[b]{2}{*}{$p$} \\
\hline & N & $\%$ & $\mathbf{N}$ & $\%$ & \\
\hline Tiempo de administración de & $\mathrm{N}=61$ Mediana & RIQ & RIQ & RIQ & \\
\hline corticoides (días) & 6 & 4 & 4 & 4 & $0,000^{c}$ \\
\hline Otros medicamentos: antibióticos & & & & & $0,207^{a}$ \\
\hline Sí & 60 & 98,36 & 98,36 & 93,33 & \\
\hline No & 1 & 1,64 & 1,64 & 6,67 & \\
\hline Total & 61 & 100,00 & 100,00 & 100,00 & \\
\hline
\end{tabular}

aPrueba exacta de Fisher // ' PPrueba Chi-cuadrado // cPrueba de U Mann-Whitney

Nota: Los estadísticos del tiempo de enoxaparina fue en base a los que recibieron el medicamento.

El uso de metilprednisolona en pulsos a dosis altas (500 mg a $1 \mathrm{gr} /$ día) aumentó en cinco veces la probabilidad de fallecer en comparación a los que usaron otros corticoides (OR ajustado=5,18 IC:1,94-13,83). Mientras que sobre la administración de dexametasona (4-6 mg/día) no se encontró asociación estadísticamente significativa con la mortalidad $(p=0,138)$, el promedio de tiempo de administración de dexametasona fue mayor en sobrevivientes que en fallecidos $(p=0,003)$ (Tabla 2).

Tabla 2. Administración de corticoides y mortalidad en pacientes con cuadro severo de COVID-19 en el Hospital Nacional Alberto Sabogal Sologuren

\begin{tabular}{|c|c|c|c|c|c|c|c|}
\hline \multirow{3}{*}{ Corticoides } & \multicolumn{4}{|c|}{ Mortalidad } & \multirow[b]{3}{*}{$p$} & \multirow[b]{3}{*}{ OR crudo [IC] } & \multirow[b]{3}{*}{ OR ajustadod [IC] } \\
\hline & \multicolumn{2}{|c|}{ sí } & \multicolumn{2}{|c|}{ NO } & & & \\
\hline & N & $\%$ & $\mathrm{~N}$ & $\%$ & & & \\
\hline \multicolumn{8}{|l|}{ Dexametasona } \\
\hline Sí & 37 & 60,66 & 44 & 73,33 & $0,138^{a}$ & & \\
\hline No & 24 & 39,34 & 16 & 26,67 & & - & \\
\hline Tiempo de & $\mathrm{N}=37$ & $\pm \mathrm{DE}$ & $\mathrm{N}=44$ & & & & \\
\hline dexametasona & Media & 3,27 & Media & $\pm \mathrm{DE}$ & & & \\
\hline \multicolumn{8}{|l|}{ (días) } \\
\hline & 7,43 & & 9,34 & 2,78 & $0,003^{b}$ & & \\
\hline Tiempo de & $N=37$ & & $N=44$ & & & & \\
\hline dexametasona & 25 & & & & & & \\
\hline$<10$ días & 12 & 67,57 & 21 & 47,73 & $0,073^{a}$ & & \\
\hline$\geq 10$ días & & 32,43 & 23 & 52,27 & & & \\
\hline \multicolumn{8}{|l|}{ Metilprednisolona } \\
\hline Sí & 23 & 37,70 & 7 & 11,67 & $0,001^{\mathrm{a}}$ & 4,58 & 5,18 \\
\hline No & 38 & 62,30 & 53 & 88,33 & & {$[1,78-11,77]$} & {$[1,94-13,83]$} \\
\hline Tiempo de & $N=23$ & RIQ & $N=7$ & RIQ & & & \\
\hline metilprednisolona & Mediana & & Mediana & & & & \\
\hline \multicolumn{8}{|l|}{ (días) } \\
\hline 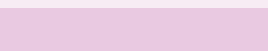 & 5 & 3 & 6 & 2 & $1,000^{c}$ & & \\
\hline
\end{tabular}

aPrueba Chi-cuadrado / / ' Prueba t-student / / cPrueba de U Mann-Whitney // dOR ajustado a edad y presencia de alguna comorbilidad. Nota: Los estadísticos del tiempo de dexametasona y metilprednisolona fue en base a los que recibieron el medicamento. 
En la figura 1 se observa que el $48,80 \%$ de pacientes fallecidos recibieron azitromicina por un tiempo mayor a 5 días, en contraste con el $11,10 \%$ de los supervivientes que recibieron el mismo tratamiento. Esto constituyó un factor asociado importante, ya que administrar este fármaco por más de 5 días aumentó en siete veces la probabilidad de fallecer, en comparación a los que recibieron el fármaco por menos tiempo (OR ajustado =7,14 IC:2,22-22,99).

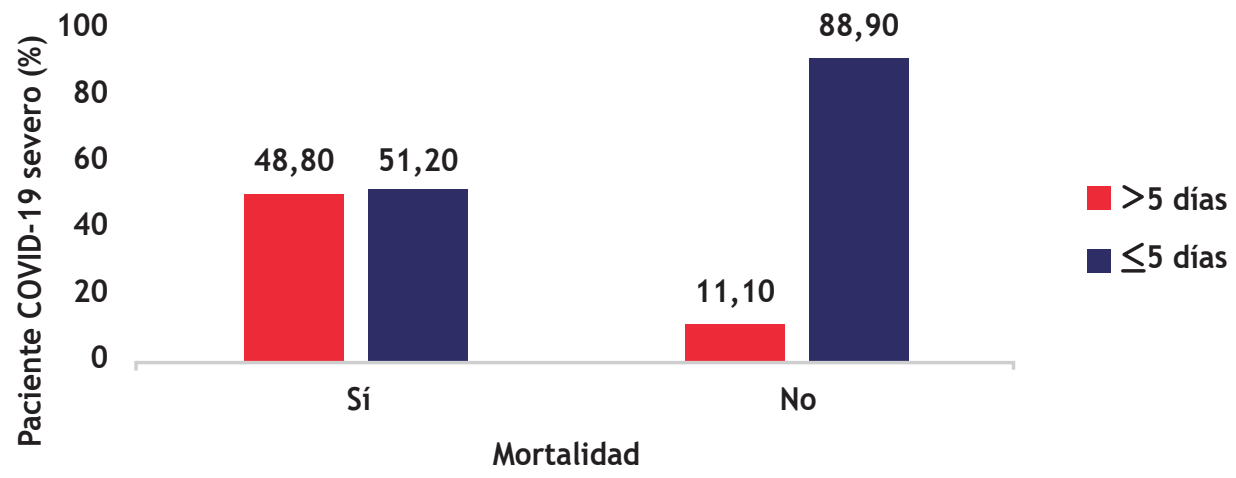

Figura 1. Uso de azitromicina mayor de 5 días y mortalidad en pacientes con cuadro severo de COVID-19 en el Hospital Nacional Alberto Sabogal Sologuren

Prueba exacta de Fisher: $\mathrm{p}=0,000$ // OR crudo: 7,61 [2,28-25,46]; OR ajustado a edad y presencia de alguna comorbilidad OR ajustado: 7,14 [2,22-22,99]. Nota: Datos analizados solo con base en los pacientes que recibieron azitromicina durante la hospitalización ( $\mathrm{N}=77)$. Tiempo de administración de azitromicina, media: 4,48 y DE:1,95 días, valor mínimo:1 y valor máximo:11 días.

No hubo asociación entre ventilación mecánica y mortalidad $(p=0,207)$. El tiempo de estancia hospitalaria fue mayor en pacientes que sobrevivieron en comparación a los fallecidos $(p=0,000)$. predictiva de mortalidad de 73,06\% para los pacientes del estudio conformado por la edad del paciente (años), administración de azitromicina mayor a 5 días y administración de metilprednisolona a dosis altas (Tabla 3).

Se construyó un modelo multivariado con probabilidad

Tabla 3. Modelo multivariado predictor de mortalidad en pacientes con cuadro severo de COVID-19 en el Hospital Nacional Alberto Sabogal Sologuren

\begin{tabular}{|lccccc|}
\hline Predictores & $B$ & $p$ & OR & \multicolumn{2}{c|}{ IC95\% } \\
& & & & Inferior & Superior \\
\hline Edad del paciente (años) & 0,036 & 0,019 & 1,04 & 1,01 & 1,07 \\
\hline Azitromicina > 5 días & 1,597 & 0,009 & 4,94 & 1,49 & 16,40 \\
\hline Administración de metilprednisolona & 1,278 & 0,013 & 3,59 & 1,30 & 9,89 \\
\hline Constante $(a)$ & $-2,848$ & 0,006 & 0,06 & 0,00 & 0,43 \\
\hline
\end{tabular}

Nota: Prueba de bondad de ajuste, prob $>X^{2}=0,000$, pseudo $R 2=0,158$. La probabilidad de predicción del modelo fue de $73,06 \%$ (Prob $\left.>X^{2}=0,731\right)$, con un área bajo la curva ROC igual a 0,756

La fórmula predictiva del modelo es

p: Probabilidad de morir

$$
\ln \left[\frac{p}{1-p}\right]=-2,848+0,036(x 1)+1,597(x 2)+1,278(x 3)
$$

x1: Edad del paciente (años)

X2: Administración de azitromicina mayor a 5 días

x3: Administración de metilprednisolona 


\section{DISCUSIÓN}

En esta investigación estudiamos a un grupo de pacientes que presentó un cuadro severo de COVID-19 y encontramos que la edad mayor a 60 años incrementó dos veces la probabilidad de fallecer, en comparación con pacientes más jóvenes. Otros estudios también coinciden en que la edad avanzada está asociada a una mayor mortalidad: la investigación de Li et al. refiere que tener de 65 años a más es un factor de riesgo de mortalidad $(p=0,021: \mathrm{HR}=1,72)^{(10)}$.

Como esquemas terapéuticos hospitalarios, todos los pacientes recibieron corticoides, y la mayoría, también enoxaparina y antibioticoterapia. La hidroxicloroquina, ivermectina y tocilizumab fueron medicamentos muy poco utilizados.

Los corticoides empleados con mayor frecuencia fueron dexametasona (4-6 mg/día) y metilprednisolona a dosis altas (500 mg a $1 \mathrm{gr}$ por día). Encontramos que la metilprednisolona incrementó en 5 veces la probabilidad de fallecer, ajustado a la edad y presencia de comorbilidad. $\mathrm{Li}$ et al. mencionan que las altas dosis de corticoides incrementan el riesgo de morir $(p=0,000 ; \mathrm{HR}=3,50){ }^{(10)}$. La Organización Mundial de la Salud (OMS) recomienda corticoterapia exclusiva en pacientes con cuadros severos: $6 \mathrm{mg} /$ día de dexametasona o su equivalente, $32 \mathrm{mg} /$ día de metilprednisolona, cantidades que son seguras para el paciente ${ }^{(14)}$ y que son mayores en la normativa peruana vigente ${ }^{(9)}$.

Edalatifard et al. concluyeron que la metilprednisolona es efectiva para pacientes hospitalizados con COVID-19 severa, que presentan menor frecuencia de mortalidad y mayor tiempo de sobrevida ${ }^{(15)}$; sin embargo, la dosis utilizada en el ensayo clínico fue de $250 \mathrm{mg} /$ día, que es menor a la dosis encontrada en esta investigación. Corral-Gudino et al. encontraron también que la metilprednisolona logró una disminución de riesgo para mortalidad en pacientes con neumonía COVID-19 (40 mg/12 h por 3 días, con una reducción por 3 días siguientes) ${ }^{(16)}$. Salton et al. refieren un resultado similar de los efectos beneficiosos de la administración prolongada de dosis bajas de metilprednisolona en pacientes con COVID-19 severo ${ }^{(17)}$.

La evidencia actual brinda una base para el uso de dosis bajas de metilprednisolona y difiere de nuestro hallazgo. La asociación de este medicamento con el desenlace de mortalidad puede estar relacionado con los efectos adversos de las dosis altas; por tanto, su administración debe realizarse con cautela.

La azitromicina ha sido promovida por la normativa peruana en dosis de $500-250 \mathrm{mg} /$ día por cinco días ${ }^{(11)}$. Sin embargo, en el presente estudio encontramos mayor tiempo de administración; su uso por más de cinco días incrementó en siete veces la probabilidad de fallecer (ajustado a edad y presencia de comorbilidad). Existe información sobre el posible efecto sinérgico antiviral en estos pacientes cuando se combina con hidroxicloroquina, cloroquina, entre otros ${ }^{(18)}$. Aunque no hay evidencia clara de los beneficios de la azitromicina en el tratamiento de la COVID-19, el tiempo de duración del fármaco, solo o en combinación con otros, no es mayor a los cinco días ${ }^{(19,20)}$. Respecto a la seguridad del fármaco, los efectos adversos que pueden ocurrir son la prolongación del intervalo QT (el más severo) ${ }^{(21)}$, la muerte cardiovascular y las arritmias ${ }^{(22,23)}$.

Planteamos la hipótesis de asociación entre un mayor tiempo de administración de azitromicina y mortalidad en los pacientes con COVID-19 severo porque esta enfermedad tiene un impacto negativo sobre la salud cardíaca que se exacerba en los cuadros graves (24). Si a esto sumamos la polifarmacia y un mayor tiempo de administración de medicamentos cardiotóxicos, como la azitromicina, puede ocurrir un efecto sinérgico que compromete la actividad cardíaca y el cuadro del paciente de manera considerable. Los pacientes estudiados no tuvieron evaluaciones cardíacas de rutina durante su hospitalización, debido a la falta de equipos de electrocardiograma, por lo que no se podría aseverar el daño cardíaco. Con base en los resultados, recomendamos un mayor cuidado en el uso y tiempo de administración de la azitromicina, sobre todo, en pacientes con cuadros severos de COVID-19.

Entre las limitaciones del estudio, podemos mencionar que los datos fueron recolectados de una fuente documental, y tanto el llenado de la historia clínica como el manejo del paciente fueron realizados por personal de salud ajeno a la investigación; por tanto, existen datos poco verificables, como el IMC o el tratamiento previo empleado, por no consignarse la fuente de donde se obtuvo (familiar o paciente con COVID-19 severo). Para el cálculo de la muestra se tomó la prevalencia de uno de los factores más importantes, la hipertensión arterial, pero no para todos los factores estudiados, ello implica que el poder de la prueba podría no ser suficiente para todos los factores considerados.

Para investigaciones posteriores, sugerimos analizar otras variables asociadas a mortalidad, como el grado de compromiso pulmonar, según los estudios de imágenes y marcadores bioquímicos de severidad. Recomendamos también desarrollar investigaciones de diseño de cohortes o ensayos clínicos relacionados a los tratamientos, en los que se analice la efectividad y la seguridad de los medicamentos empleados.

En conclusión, los pacientes con un cuadro severo de COVID-19 que recibieron metilprednisolona en dosis altas tuvieron mayor probabilidad de fallecer, al igual que los 
que recibieron azitromicina por más de cinco días. Otro factor asociado a la mortalidad fue la edad mayor a 60 años. El modelo multivariado construido con los datos del estudio tiene una capacidad predictiva de mortalidad de $75,60 \%$ y analiza la edad del paciente, administración de azitromicina mayor a 5 días y administración de metilprednisolona a dosis altas.

Contribuciones de los autores: MLI, RG, BG y KC participaron en la contribución de la idea original y elaboración del proyecto del manuscrito; MLI y RG recolectaron los datos; BG y KC analizaron e interpretaron los datos; MLI, RG, BG y KC realizaron la redacción y aprobación de la versión final, además todos los autores se hacen responsables del contenido del artículo.

Agradecimientos: A la Dra. Diana Rodríguez Hurtado por su apoyo en el diseño metodológico.

Fuentes de financiamiento: Este artículo ha sido financiado por los autores.

Conflictos de interés: Los autores declaran no tener ningún conflicto de interés.

\section{REFERENCIAS BIBLIOGRÁFICAS}

1. Organización Mundial de la Salud. COVID-19: cronología de la actuación de la OMS [Internet]. 2020. Disponible en: https://www. who.int/es/news-room/detail/08-04-2020-who-timeline---covid-19

2. Organización Mundial de la Salud. Alocución de apertura del Director General de la OMS en la rueda de prensa sobre la COVID-19 celebrada el 11 de marzo de 2020 [Internet]. 2020. Disponible en: https: / /www. who.int/es/dg/speeches/detail/who-director-general-s-openingremarks-at-the-media-briefing-on-covid-19---11-march-2020

3. World Health Organization. Coronavirus disease 2019 (COVID-19) Situation Report-101 [Internet]. 2020. Disponible en: https:// www. who.int/docs/default-source/coronaviruse/situationreports/20200430-sitrep-101-covid-19.pdf?sfvrsn=2ba4e093_2

4. Ministerio de Salud. Covid 19 en el Perú - Sala Situacional COVID-19 Perú [Internet]. Lima; 2020. Disponible en: https://covid19.minsa. gob.pe/sala_situacional.asp

5. Feng Y, Ling Y, Bai T, Xie Y, Huang J, Li J, et al. COVID-19 with different severity: a multi-center study of clinical features. Am J Respir Crit Care Med. 2020; 201(11): 1380-8.

6. Zhou F, Yu T, Du R, Fan G, Liu Y, Liu Z, et al. Clinical course and risk factors for mortality of adult inpatients with COVID-19 in Wuhan, China: a retrospective cohort study. Lancet. 2020; 395(10229): 1054-62.

7. Xu X, Han M, Li T, Sun W, Wang D, Fu B, et al. Effective treatment of severe COVID-19 patients with tocilizumab. Proc Natl Acad Sci. 2020; 117(20): 10970-5.

8. Mahevas M, Tran V-T, Roumier M, Chabrol A, Paule R, Guillaud C, et al. No evidence of clinical efficacy of hydroxychloroquine in patients hospitalized for COVID-19 infection with oxygen requirement: results of a study using routinely collected data to emulate a target trial. medRxiv. 2020.

9. Ministerio de Salud. RM N ${ }^{\circ}$ 240-2020-Minsa. Modificación del Documento Técnico: Prevención, Diagnóstico y Tratamiento de personas afectas por COVID-19 en el Perú [Internet]. Lima; 2020. Disponible en: http://sph-peru.org/wp-content/uploads/2020/05/ RM-240-2020-MINSA.PDF.pdf

10. Li X, Xu S, Yu M, Wang K, Tao Y, Zhou Y, et al. Risk factors for severity and mortality in adult COVID-19 inpatients in Wuhan. J Allergy Clin Immunol. 2020; 146(1): 110-8.

11. Ministerio de Salud. RM N ${ }^{\circ}$ 139-2020-Minsa. Documento Técnico Prevención y atención de personas afectadas COVID-19 en el Perú [Internet]. Lima; 2020. Disponible en: https://cdn.www.gob.pe/ uploads/document/file/574295/resolucion-ministerial-139-2020MINSA.PDF

12. Ministerio de Salud. RM N ${ }^{\circ}$ 270-2020-Minsa. Modificación del Documento Técnico: Prevención, Diagnóstico y Tratamiento de personas afectas por COVID-19 en el Perú [Internet]. Lima; 2020. Disponible en: https://cdn.www.gob.pe/uploads/document/ file/694719/RM_270-2020-MINSA.PDF

13. Ministerio de Salud. RM N ${ }^{\circ}$ 283-2020-Minsa. Modificación del Documento Técnico: Prevención, Diagnóstico y Tratamiento de personas afectas por COVID-19 en el Perú [Internet]. Lima; 2020. Disponible en: https://cdn.www.gob.pe/uploads/document/ file/710132/RM_283-2020-MINSA.PDF

14. Organización Mundial de la Salud. Corticosteroides for COVID-19. Living Guidance [Internet]. 2020. Disponible en: https://www.who. int/publications/i/item/WHO-2019-nCoV-Corticosteroids-2020.1

15. Edalatifard M, Akhtari M, Salehi M, Naderi Z, Jamshidi A, Mostafaei $\mathrm{S}$, et al. Intravenous methylprednisolone pulse as a treatment for hospitalised severe COVID-19 patients: results from a randomised controlled clinical trial. Eur Respir J. 2020; 56(6): 2002808.

16. Corral L, Bahamonde A, Revillas FA, Gomez-Barquero J, AbadiaOtero J, Garcia-Ibarbia C, et al. GLUCOCOVID: A controlled trial of methylprednisolone in adults hospitalized with COVID-19 pneumonia. medRxiv. 2020.

17. Salton F, Confalonieri P, Meduri GU, Santus P, Harari S, Scala R, et al. Prolonged low-dose methylprednisolone in patients with severe COVID-19 pneumonia. Open Forum Infect Dis. 2020; 7(10): ofaa421.

18. Sultana J, Cutroneo PM, Crisafulli S, Puglisi G, Caramori G, Trifirò G. Azithromycin in COVID-19 patients: pharmacological mechanism, clinical evidence and prescribing guidelines. Drug Saf. 2020; 1-8.

19. Gautret P, Lagier J-C, Parola P, Hoang VT, Meddeb L, Sevestre J, et al. Clinical and microbiological effect of a combination of hydroxychloroquine and azithromycin in 80 COVID-19 patients with at least a six-day follow up: a pilot observational study. Travel Med Infect Dis. 2020; 34: 101663.

20. Arshad S, Kilgore P, Chaudhry ZS, Jacobsen G, Wang DD, Huitsing $\mathrm{K}$, et al. Treatment with hydroxychloroquine, azithromycin, and combination in patients hospitalized with COVID-19. Int J Infect Dis. 2020; 97: 396-403.

21. Zeng L, Xu P, Choonara I, Bo Z, Pan X, Li W, et al. Safety of azithromycin in pediatrics: a systematic review and meta-analysis. Eur J Clin Pharmacol. 2020; 76(12): 1709-21.

22. Zaroff JG, Cheetham TC, Palmetto N, Almers L, Quesenberry C, Schneider J, et al. Association of azithromycin use with cardiovascular mortality. JAMA Netw Open. 2020; 3(6): 208199.

23. U. S. Food and Drug Administration. FDA Drug Safety Communication: Azithromycin (Zithromax or Zmax) and the risk of potentially fatal heart rhythms [Internet]. 2019. Disponible en: https: / /www.fda.gov/ drugs/drug-safety-and-availability/fda-drug-safety-communicationazithromycin-zithromax-or-zmax-and-risk-potentially-fatal-heart

24. Kalra RS, Tomar D, Meena AS, Kandimalla R. SARS-CoV-2, ACE2, and hydroxychloroquine: cardiovascular complications, therapeutics, and clinical readouts in the current settings. Pathogens. 2020; 9(7): 546. 


\section{Correspondencia:}

Karen Elizabeth Campos Correa

Dirección: Jr. Gabriel Aguilar, 10 de Octubre.

San Juan de Lurigancho. Lima, Perú.

Teléfono: 959351134

Correo electrónico: karen.campos@upch.pe

\section{Enviado: 07 de enero de 2021}

Evaluado: 18 de enero de 2021

Aprobado: 04 de febrero de 2021

(c) La revista. Publicado por Universidad de San Martín de Porres, Perú. (cc) Br Licencia de Creative Commons Artículo en acceso abierto bajo términos de Licencia Creative Commons Atribución 4.0 Internacional. (http://creativecommons.org/licenses/by/4.0/)

ORCID iDs

Manuel K. Llaro-Sánchez

Ronald N. Guzman-Ramos

Bernardo E. Gamarra-Villegas

Karen E. Campos-Correa
(1) https: / / orcid.org/0000-0002-9282-8301

(1) https: / / orcid.org/0000-0002-2055-0697

(-) https: / / orcid.org/0000-0002-4680-1861

(- https: / / orcid.org/0000-0001-6664-2557 